\title{
A Contrast Nephropathy in a Preterm Infant Following Preoperative Embolization of Giant Sacrococcygeal Teratoma
}

\author{
Byong Sop Lee, M.D., Ph.D. \\ Department of Pediatrics University \\ of Ulsan, College of Medicine, Asan \\ Medical Center, Seoul, Korea \\ Corresponding author: \\ Byong Sop Lee, M.D., Ph.D. \\ Department of Pediatrics, Asan Medical \\ Center Children's Hospital, University \\ of Ulsan College of Medicine, 388-1 \\ Poongnap-dong, Songpa-gu, Seoul \\ 05505, South Korea \\ Tel: +82-2-3010-3929 \\ Fax: +82-2-3010-6978 \\ E-mail:mdleebs@amc.seoul.kr
}

Received: 13 January 2017

Revised: 10 March 2017

Accepted: 15 March 2017
Newborn infants with huge and highly vascular sacrococcygeal teratoma (SCT) are frequently subjected to renal hypoperfusion secondary to high-output cardiac failure. Any underlying renal dysfunction is a significant risk factor for the development of contrast-induced nephropathy (CIN). However, reports on CIN in infants are rare. I report here a case of a premature infant born at 28 weeks and 3 days of gestation with a huge SCT who survived preoperative embolization and surgical resection but presented with persistent non-oliguric renal failure that was suggestive of CIN. During radiological intervention, a contrast medium had been administered at about 10 times the manufacturer-recommended dose for pediatric patients. Despite hemodynamic stabilization and normalization of urine output immediately following surgery, the patient's serum creatinine and cystatin-C levels did not return to baseline until 4 months after birth. No signs of reflux nephropathy were observed in follow-up imaging studies. Dosing guidelines for the use of a contrast medium in radiological interventions should be provided for infants or young patients.

Key words: Acute kidney injury, Contrast-induced nephropathy, Sacrococcygeal teratoma, Premature infant, Therapeutic embolization

\section{Introduction}

Acute kidney injury (AKI) is a frequently encountered problem in premature infants, especially during the early neonatal period. In premature infant, one of the main pathogenesis of AKI is renal hypoperfusion secondary to cardiovascular compromise. Several perinatal conditions including perinatal distress and heart failure secondary to hemodynamically significant patent ductus arteriosus are common causes of AKI in premature infants ${ }^{1)}$.

Sacrococcygeal teratoma (SCT) is the most common neoplasm in neonates demonstrating an estimated incidence of 1 of 40,000 births and the complete surgical excision is the primary treatment of choice ${ }^{2)}$. A subgroup of infants with huge and highly vascular SCT, however, frequently presents with fetal hydrops and high-output heart failure and is at high risk of mortality and renal morbidity. Several preoperative procedures that decrease the blood supply to the tumor and thereby stabilize the patient's hemodynamic condi- 
tion and minimize intraoperative bleeding have been proposed. These include radiofrequency ablation, laparoscopic ligation and angiographic embolization of the feeding arteries $^{2,3)}$. Although a few cases of successful preoperative embolization using contrast media have been reported even in extremely premature infants ${ }^{3-5)}$, no detailed data have been reported regarding the short- and long-term outcomes of premature infants following radiological intervention using contrast media.

Here, I present the case of a premature infant with a huge SCT who survived preoperative embolization and mass excision but subsequently developed AKI that persisted until 4 months after birth. An overdose of contrast media was suspected as a significant contributor to the development of non-oliguric AKI.

\section{Case report}

A large sacral mass was detected in a fetus by ultrasound at 23 weeks of gestation. The prenatal targeted ultrasound performed at 1 week later revealed a $96 \times 85 \mathrm{~mm}$ SCT with high vascularity that was invading the pelvic cavity. At 24 weeks and 3 days of gestation, ultrasound-guided laser ablation of the mass was attempted but failed. The size of the mass increased and severe polyhydramnios developed. The mother was hospitalized at 27 and 4 days of gestation because of premature rupture of membrane and chest discomfort. She was treated with antibiotics and a single course ( 2 doses) of antenatal betamethasone. At 28 weeks and 3 days of gestation, a boy was delivered via an elective cesarean section with a birth weight of 2,940 g including the huge pelvic mass (Fig 1). The infant was intubated for respiratory distress in the delivery room and managed with positive pressure ventilation. The Apgar score was 3 and 5 at 1 minute and 5 minutes, respectively. On admission, there was massive bleeding from the ulceration site on the surface of the tumor and it was controlled by sutures. Severe anemia and the feature of disseminated intravascular coagulation were detected and corrected using blood products. Surfactant was also administered and ventilator care was required to maintain adequate ventilation and oxygenation. The infant was managed with several bolus infusions and inotropics, including dopamine and dobutamine due to systemic hypotension. On the second day of birth, abdominal and pelvic CT revealed a huge hypervascular mass with intrapelvic extension to the posterior aspect of the bladder. He was anuric for the first 2 days of life. On the third day of birth, embolization of the tumor was performed through the left common carotid artery. A 2.2-Fr probe was guided via the distal abdominal aorta to the bilateral internal iliac arteries and one lumbar artery. Occlusion of the feeding arteries was performed using gel forms, polyvinyl alcohol and metal coils. However, the intervention process was stopped because of the patient's unstable condition and the determination of a contrast media overdose (Iodixanol, Visopaque ${ }^{\circledast} 270$, GE Healthcare, Cork, Island; a total of $25 \mathrm{~mL}$ was diluted with the same volume of saline). Post-embolization angiography still showed some remaining flow to the mass (Fig. 2). Immediately after the procedure, the patient's blood pressure increased and the dose of inotropics was tapered. He first voided at 12 hours after embolization. On the fourth day of birth, complete mass excision, including the pelvic portion of the mass, was performed. However, multiple blood transfusions were needed due to massive bleeding during the operation. He experienced cardiac arrest 3 times, presumably due to transfusion-associated hypocalcemia (lowest ionized calcium level: $0.20 \mathrm{mmol} / \mathrm{L}$ ) and was resuscitated using intermittent cardiac compression $(<1$ minute for each duration) and multiple calcium infusions. The excised mass weighed $1,880 \mathrm{~g}(16.0 \times 11.0 \times 10.5 \mathrm{~cm})$. Pathologic

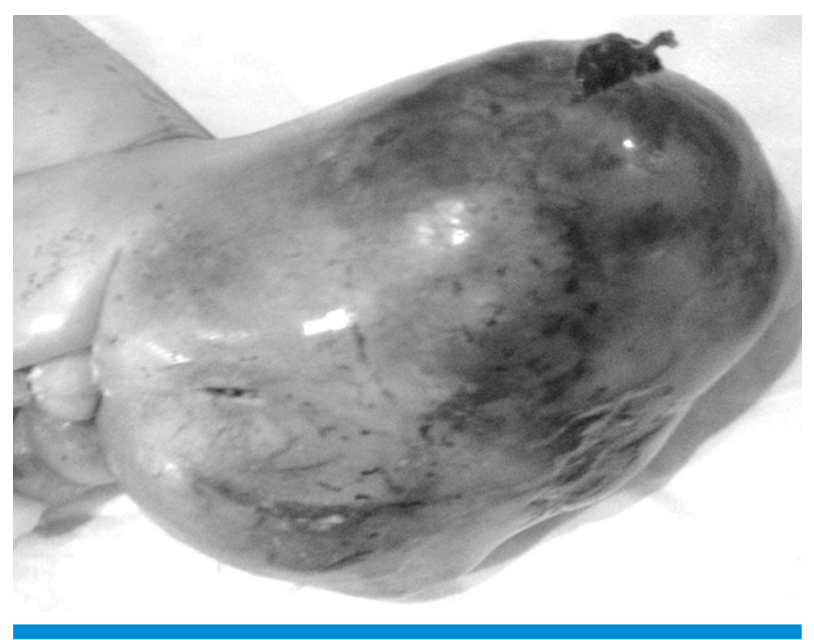

Fig. 1. The patient born at 28 weeks and 3 days of gestational age with a huge sacrococcygeal teratoma. Note significant edema in the extremities and trunk and the huge mass with ulceration on the surface. 
examination verified that the mass was a grade 3 immature teratoma. Urine output normalized on the day of the operation and thereafter, although urinary dribbling was observed. Neurogenic bladder was clinically suspected but urodynamic studies were not performed. However, neither the serum creatinine nor the cystatin-C levels normalized during the hospitalization period or by the time of the last follow-up examination (Fig. 3). The patient was weaned off

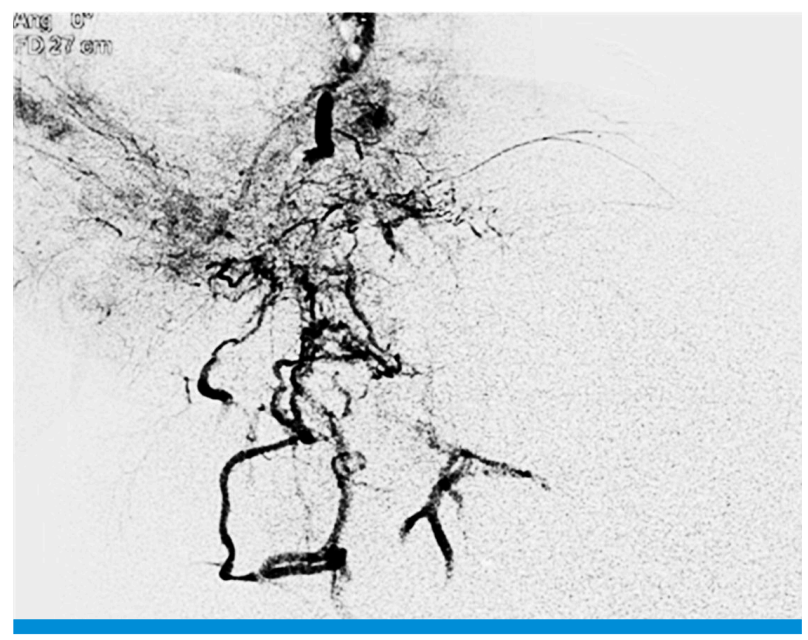

Fig.2. On post-embolization angiography, some flow to the mass via the branches of the distal external ileac artery and common femoral artery can be seen. the ventilator on 10th day of birth. No signs of hypoxicischemic brain injury were found on serial ultrasounds and brain magnetic resonance imaging performed at termequivalent age. He was discharged at 9 weeks of life (corrected age of 37 weeks and 4 days). Serial kidney ultrasound revealed persistent increases in the echogenecity of both kidneys, but they were of normal size without evidence of hydronephrosis. By 2 years of corrected age, the patient demonstrated rapid catch-up growth but development was significantly delayed for his corrected age. Neurogenic bladder persisted. There was no evidence of tumor recurrence on serial pelvic MRIs.

\section{Discussion}

Although the causes of prolonged AKI in our patient were not clear and might be multifactorial, the clinical course of our patient was suggestive of contrast-induced nephropathy (CIN) that resulted from an overdose of contrast media. CIN is generally defined as an increase in the serum creatinine level to $>25 \%$ or $0.5 \mathrm{mg} / \mathrm{dL}$ over the baseline within 3 days following the administration of contrast media ${ }^{6)}$ and commonly manifests as a non-oliguric renal

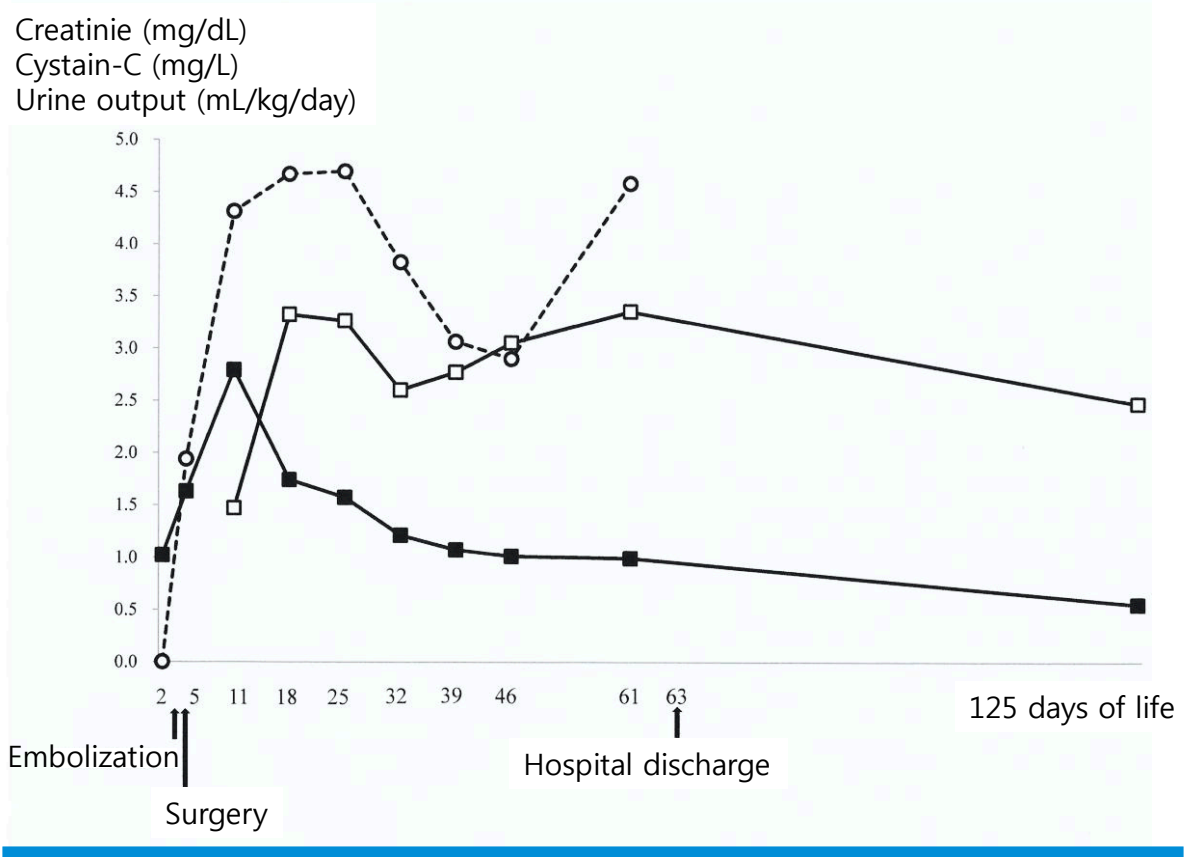

Fig.3. Serial changes in serum creatinine (closed rectangle), cystatin-C (open rectangle) and urinary output (open circle). 
failure $^{7}$. In this case, urine output was normalized following the surgery despite the prolonged decline in renal function as evidenced by the persistent increase in the serum creatinine level. Serum cystatin-C, another reliable marker of renal injury even in preterm infants ${ }^{8}$, also did not normalize until the last follow-up examination. Among the many known risk factors of CIN, preexisting impairment of renal function is the most well-established ${ }^{7}$. In addition to immature kidneys with a low glomerular filtration rate, perinatal conditions that develop in newborn infants with huge SCT, including "steal phenomenon" by the hypervascular tumor mass and unstable hemodynamic conditions, may contribute to the development of renal impairment. It seems unlikely that post-renal factors, the most common postoperative complications in the survivors of huge SCT, conferred the significant risk of development of AKI in our patient, because there was no apparent evidence of reflux or obstructive nephropathy on the follow-up examination.

The administered dose of contrast media is also a definite risk factor associated with the development of CIN. Although a large volume and multiple injections of contrast media have been reported in association with CIN in animal and human studies ${ }^{9}$, no cut-off values have been established. The dose of the administered contrast media delivered to our patient $(25 \mathrm{~mL}$ : approximately $8.5 \mathrm{~mL} / \mathrm{kg}$ of body weight including the tumor) is similar to the suggested threshold of the total dose of a low-osmolarity contrast media $(30 \mathrm{~mL})$ that is associated with the markedly increased incidence of CIN in adults ${ }^{10}$. The manufacturer recommends that the intra-arterial dose of iodixanol administered to pediatric patients $>1$ year of age be $1-2 \mathrm{~mL} /$ $\mathrm{kg}$, and the total dose should not exceed $4 \mathrm{~mL} / \mathrm{kg}^{11)}$. Although the epidemiology of CIN in pediatric populations has not been well studied, the incidence of transient CIN within the recommended range has been reported as 10.6 $\%$ in pediatric patients who have received the intravenous administration of the same agent ${ }^{12)}$. In contrast, another pediatric study on the contrast dose during cardiac catheterization reported extremely low adverse effects even when a large volume of a different kind of contrast media (Optiray $350^{\circ}$, Mallinkrodt, St Louis, Missouri, USA) was administered at a dose of $\geq 6 \mathrm{~cm}^{3} / \mathrm{kg}^{13)}$.

It is still uncertain if even the complete occlusion of the feeding arteries could have reduced the risk of significant bleeding during the surgery because the chance of internal tumor bleeding and disseminated intravascular coagulation always exist, both prenatally and postnatally. A recent study reported the successful surgical resection of a giant SCT in a premature infant who was born at 30 weeks and 3 days of gestation following preoperative embolization ${ }^{5}$. Unfortunately, life-threatening cardiac arrest also could not be avoided, presumably due to hemolysis and transfusion-associated hyperkalemia. Our patient, who was born even more premature and with a greater tumor to birth weight ratio (0.63) than the previously reported case, also experienced a few life-threatening events during the operation. In recent 2 large-center studies, a survival rate of about $50 \%$ was reported following surgical resection even in premature infants with large SCT who did not receive preoperative management including embolization ${ }^{14,15)}$. A large body of evidence is needed to provide optimal treatment guidelines for giant SCT in neonates.

In conclusion, here I report the case of a premature infant with suspected CIN which was possibly related to an overdose of contrast media used for the preoperative embolization of a huge SCT. Further data on renal outcomes are needed to devise optimal dosing strategies for newborn infants receiving radiologic intervention, especially those at high risk of developing AKI.

\section{Conflicts of interest}

No potential conflict of interest relevant to this article was reported.

\section{References}

1. Cataldi L, Leone R, Moretti U, De Mitri B, Fanos V, Ruggeri L, et al. Potential risk factors for the development of acute renal failure in preterm newborn infants: a case-control study. Arch Dis Child Fetal Neonatal Ed 2005;90:F514-9.

2. Hedrick HL, Flake AW, Crombleholme TM, Howell LJ, Johnson MP, Wilson RD, et al. Sacrococcygeal teratoma: prenatal assessment, fetal intervention, and outcome. J Pediat Surg 2004;39: 430-8.

3. Cowles RA, Stolar CJ, Kandel JJ, Weintraub JL, Susman J, Spigland NA. Preoperative angiography with embolization and radiofre- 
quency ablation as novel adjuncts to safe surgical resection of a large, vascular sacrococcygeal teratoma. Pediatr Surg Int 2006; 22:554-6.

4. Ding J, Chen Q, Stone P. Percutaneous laser photocoagulation of tumour vessels for the treatment of a rapidly growing sacrococcygeal teratoma in an extremely premature fetus. J Matern Fetal Neonatal Med 2010;23:516-8.

5. Lahdes-Vasama TT, Korhonen PH, Seppanen JM, Tammela OK, Iber T. Preoperative embolization of giant sacrococcygeal teratoma in a premature newborn. J Pediatr Surg 2011;46:e5-8.

6. Karcaaltincaba M, Oguz B, Haliloglu M. Current status of contrastinduced nephropathy and nephrogenic systemic fibrosis in children. Pediatr Radiol 2009;39 Suppl 3:382-4.

7. Gleeson TG, Bulugahapitiya S. Contrast-induced nephropathy. Am J Roentgenol 2004;183:1673-89.

8. Finney H, Newman DJ, Thakkar H, Fell JM, Price CP. Reference ranges for plasma cystatin $C$ and creatinine measurements in premature infants, neonates, and older children. Arch Dis Child 2000:82:71-5.

9. Cigarroa RG, Lange RA, Williams RH, Hillis LD. Dosing of contrast material to prevent contrast nephropathy in patients with renal disease. Am J Med 1989;86:649-52.

10. Manske CL, Sprafka JM, Strony JT, Wang Y. Contrast nephropathy in azotemic diabetic patients undergoing coronary angiography. Am J Med 1990;89:615-20.

11. VISIPAQUE (iodixanol) Injection. http://md.gehealthcare.com/ pifiles/Visipaque.pdf. [Accessed on 2 August 2012].

12. Zo'o M, Hoermann M, Balassy C, Brunelle F, Azoulay R, Pariente D, et al. Renal safety in pediatric imaging: randomized, doubleblind phase IV clinical trial of iobitridol 300 versus iodixanol 270 in multidetector CT. Pediatr Radiol 2011:41:1393-400.

13. Senthilnathan S, Gauvreau K, Marshall AC, Lock JE, Bergersen L. Contrast administration in pediatric cardiac catheterization: dose and adverse events. Catheter Cardiovasc Interv 2009;73:814-20.

14. Holcroft CJ, Blakemore KJ, Gurewitsch ED, Driggers RW, Northington FJ, Fischer AC. Large Fetal Sacrococcygeal Teratomas: Could Early Delivery Improve Outcome? Fetal Diagn Ther 2008; 24:55-60.

15. Roybal JL, Moldenhauer JS, Khalek N, Bebbington MW, Johnson $\mathrm{MP}$, Hedrick HL, et al. Early delivery as an alternative management strategy for selected high-risk fetal sacrococcygeal teratomas. J Pediatr Surg 2011;46:1325-32. 